\title{
Hydraulic Water Permeability and Transepithelial Voltage in the Isolated Perfused Rabbit Cortical Collecting Tubule Following Acute Unilateral Ureteral Obstruction
}

\author{
H. T. Campbell, E. Bello-Reuss, and S. Klahr \\ Department of Physiology and Biophysics, and Renal Division, Department of Medicine, \\ Washington University School of Medicine, St. Louis, Missouri 63110
}

\begin{abstract}
Ureteral obstruction affects the kidney's ability to conserve water and sodium. Using the isolated perfused tubule technique, we studied cortical collecting tubules (CCT) taken from rabbits subjected to a sham operation or to $4 \mathrm{~h}$ of unilateral ureteral obstruction (UUO). Tubules were perfused in the presence of an osmotic gradient directed to promote water movement from lumen to bath, and volume flux $\left(J_{v}\right)$, hydraulic water permeability $\left(L_{p}\right)$, and transepithelial voltage $\left(V_{1}\right)$ were determined. In tubules from sham-operated and UUO animals, basal (before exposure to vasopressin) $J_{v}$ and $L_{p}$ were not different from zero. After addition of $200 \mu \mathrm{U} \cdot \mathrm{ml}^{-1}$ of arginine vasopressin (aVP) to the bath, $J_{v}$ and $L_{p}$ increased to $1.64 \pm 0.23$ $\mathrm{nl} \cdot \mathrm{mm}^{-1} \cdot \mathrm{min}^{-1}$ and $127.9 \pm 19.8 \mathrm{~cm} \cdot \mathrm{s}^{-1} \cdot \mathrm{atm}^{-1} \cdot 10^{7}$, respectively, in tubules from sham-operated animals, but to only $0.27 \pm 0.09 \mathrm{nl} \cdot \mathrm{mm}^{-1} \cdot \mathrm{min}^{-1}$ and $18.8 \pm 6.2 \mathrm{~cm} \cdot \mathrm{s}^{-1} \cdot \mathrm{atm}^{-1} \cdot 10^{7}$ in tubules from UUO animals. Pretreatment with desoxycorticosterone acetate (DOCA) or indomethacin in vivo did not prevent the blunted vasopressin response seen in tubules taken from UUO animals. The $J_{v}$ and $L_{p}$ responses to the cyclic AMP (cAMP) analogue, 8-Br-cAMP, were also diminished in tubules taken from UUO animals compared with shams. $V_{1}$, measured during the basal period, was diminished in tubules from UUO kidneys $(-5.0 \pm 2.1 \mathrm{mV})$ compared with shams $(-21.9 \pm 4.1 \mathrm{mV})$, and pretreatment with DOCA did not prevent the effects of UUO on $V_{1}$. In contrast, tubules taken from animals that received indomethacin prior to UUO developed voltages not different from voltages in tubules taken from sham-operated animals $(-17.3 \pm 1.7 \mathrm{mV})$. We conclude that, although CCT from UUO animals can maintain osmotic gradients, their ability to respond to vasopressin by increasing $L_{p}$ is impaired by an intrinsic defect located at a step beyond the generation of CAMP, and that prostaglandin inhibition or DOCA pretreatment do not reverse the decreased responsiveness of $L_{p}$ to aVP. UUO also diminished $V_{1}$, and this abnormality was prevented by previous treatment with indo-
\end{abstract}

Part of this material was presented at the 16th Annual Meeting of the American Society of Nephrology and was published in abstract form in 1984. Kidney Int. 25:228. Dr. Campbell is a postdoctoral fellow of the Renal Division, Department of Medicine, Washington University School of Medicine.

Address reprint requests to Dr. Bello-Reuss, Department of Physiology and Biophysics, Washington University School of Medicine, 660 South Euclid Avenue, Box 8101, St. Louis, MO 63110. 1984.

Received for publication 3 July 1984 and in revised form 20 October

J. Clin. Invest.

(c) The American Society for Clinical Investigation, Inc.

0021-9738/85/01/0219/07 \$1.00

Volume 75, January 1985, 219-225 methacin, suggesting that prostaglandins may mediate the effect of UUO on $V_{1}$.

\section{Introduction}

Ureteral obstruction causes a number of alterations in renal function. Abnormalities in sodium and water conservation are well-known consequences of partial ureteral obstruction and of the state that follows release of complete ureteral obstruction $(1,2)$. A number of factors may play a role in the pathophysiology of this state. Extrarenal factors, such as solute and water overload and the presence of natriuretic factors retained during the period of obstruction, may contribute to diminished sodium and water reabsorption by the postobstructed kidney, as may changes in renal hemodynamics, glomerular filtration rate, and intrarenal production of hormones (2-7). The roles of these extrarenal and extratubular factors have been studied in some detail. However, it is possible that obstruction affects the function of the tubular epithelium per se, such that the normal intrinsic capacity of the tubule to reabsorb sodium and water is impaired.

Hanley and Davidson (8) studied the effects of obstruction on several segments of the rabbit nephron using the isolated perfused tubule technique. After $24 \mathrm{~h}$ of ureteral obstruction, they found decreased hydraulic water permeability in cortical collecting tubules (CCT) that had been perfused in the presence of vasopressin and then exposed to an osmotic gradient. To further investigate the effects of brief periods of unilateral ureteral obstruction (UUO) ${ }^{1}$ on the CCT, we conducted experiments to study the ability of the tubules to $(a)$ maintain an osmotic gradient in the absence of vasopressin, $(b)$ respond to vasopressin by increasing hydraulic water permeability $\left(\mathrm{L}_{\mathrm{p}}\right)$, and $(c)$ generate a transepithelial voltage $\left(V_{1}\right)$. The effects of mineralocorticoids, prostaglandins, and cyclic AMP (cAMP) on the function of the CCT after UUO was also investigated. Our results indicate that brief periods of UUO induce abnormalities in both vasopressin-induced osmotic water permeability and $V_{l}$, and that the abnormality in voltage can be prevented by pretreatment with indomethacin.

\section{Methods}

Female New Zealand white rabbits weighing 1.5-2.0 kg were fed a standard chow and given free access to water for at least 1 wk prior to study. On the day of the experiment, all animals were anesthetized

1. Abbreviations used in this paper: 8-Br-cAMP, 8-bromoadenosine 3',5'-cyclic monophosphate; CCT, cortical collecting tubule; DOCA, desoxycorticosterone acetate; $J_{v}$, volume flux; $L_{p}$, hydraulic water permeability; UUO, unilateral ureteral obstruction; $V_{i}$, perfusion rate; $V_{1}$, transepithelial voltage. 
with pentobarbital ( $25-30 \mathrm{mg} \cdot \mathrm{kg}^{-1}$ body wt given intravenously) and subjected to either UUO or a sham operation. All animals had midline lower abdominal incisions and identification of the ureter at the ureterovesical junction; in the UUO group, the ureter was ligated twice with 2-0 silk suture. After surgery the animals were allowed to recover under a heat lamp and denied access to food and water. $4 \mathrm{~h}$ after the procedure, the animals were sacrificed by decapitation, and obstructed or the sham kidney was quickly removed. $1 \mathrm{~mm}$ thick coronal slices were obtained and kept in chilled media (identical to the bathing solution) during dissection. CCT were separated from corticomedullary rays with fine forceps (Dumont no. 5, Ernest F. Fullam Inc., Schenectady, NY) under $\times \mathbf{4 0}$ magnification. CCT were identified on the basis of the junction of two or more segments to form tubules of characteristic appearance (9). Kidneys subjected to ureteral obstruction were swollen and often showed areas of hemorrhage which were avoided during dissection. After isolation, the tubule was transferred to a lucite chamber and perfused as previously described $(10,11)$. Briefly, one end of the tubule was aspirated into a holding pipette and a second smaller pipette introduced into the tubular lumen for delivery of perfusate. The other end of the tubule was aspirated into a collection pipette filled with water-equilibrated mineral oil so that collected fluid displaced the oil. The tip of the collection pipette was coated with a thin film of the insulating resin Sylgard 184 (Dow-Corning, Midland, MI). Sylgard 184 was not used in the holding pipette since in our experience voltages measured via the perfusion pipette (see below) were not affected by the presence of Sylgard. Timed collections of perfused fluid were made with precalibrated volumetric pipettes of 40$50 \mathrm{nl}$. The bathing solution consisted of (in millimolar): $\mathrm{NaCl}, 105$; $\mathrm{NaHCO}_{3}, 25 ; \mathrm{KCl}, 5 ; \mathrm{CaCl}_{2}, 1.8 ; \mathrm{MgSO}_{4}, 1.0 ; \mathrm{Na}_{2} \mathrm{HPO}_{4}, 2.5 ; \mathrm{Na}$ acetate, 10; glucose, 8.3; and alanine, 5.0. This solution was bubbled with $95 \% \mathrm{O}_{2}: 5 \% \mathrm{CO}_{2}$ at $37^{\circ} \mathrm{C}$ to $\mathrm{pH} 7.4$. The perfusate was modified from the bathing solution by lowering the $\mathrm{NaCl}$ to $42 \mathrm{mM}$ and omitting the $\mathrm{NaHCO}_{3}$, glucose, and alanine. The $\mathrm{pH}$ of the perfusate, when equilibrated with $5 \% \mathrm{CO}_{2}$ at $37^{\circ} \mathrm{C}$, was $6.13 .50 \mu \mathrm{Ci} \cdot \mathrm{ml}^{-1}$ of previously dialyzed tritiated methoxy-inulin (New England Nuclear, Boston, MA) was added to the perfusate to serve as a volume marker in the determination of net volume flux $\left(J_{v}\right)$, perfusion rate $\left(V_{i}\right)$, and $\mathrm{L}_{\mathrm{p}}$. The osmolalities of all solutions were determined before each experiment with a vapor pressure osmometer (Wescor, Inc., Logan, UT). The measured osmolalities of the bath and perfusion solutions were $290 \pm 5$ and $132 \pm 8 \mathrm{mosm} \cdot \mathrm{kg}^{-1}$, respectively (means $\pm \mathrm{SD}$ ). All experiments were conducted at $37^{\circ} \mathrm{C}$. The chamber volume was 1.0 $\mathrm{ml}$; fresh bath solution was exchanged continuously at a rate of 1-2 $\mathrm{ml} / \mathrm{min}$. $\mathbf{V}_{\mathbf{i}}$ (nanoliters per minute) was calculated as:

$\mathrm{V}_{\mathrm{i}}={ }^{3} \mathrm{H}_{0} /\left[{ }^{3} \mathrm{H}_{\mathrm{i}}\right] \cdot t$

where ${ }^{3} \mathrm{H}_{0}$ is the total number of tritium counts collected, $\left[{ }^{3} \mathrm{H}_{i}\right]$ is the concentration of counts in the perfusate solution (cpm per nanoliters), and $t$ is the time required for collection (minutes) (12). $V_{i}$ was $9.05 \pm 1.80 \mathrm{nl} \cdot \mathrm{min}^{-1}$ for the sham-operated tubules and $8.52 \pm 1.58$ $\mathrm{nl} \cdot \mathrm{min}^{-1}$ for the UUO tubules (means $\pm \mathrm{SD}$ ). Tubule lengths, measured with an eyepiece micrometer, were $1.3 \pm 0.4 \mathrm{~mm}$ for the tubules from sham-operated animals and $1.5 \pm 0.5 \mathrm{~mm}$ for the tubules of animals with UUO (means $\pm \mathrm{SD}$ ). Tubules were excluded from anlysis if defects in the tubule wall were seen by inspection under $\times 200$ magnification or if the concentration of tracer in the collected fluid was less than that in the perfused fluid by $2 \%$ or more during the basal period (absence of vasopressin), indicating leak of bath solution into the tubule lumen or collection pipette.

$\mathbf{J}_{\mathbf{v}}$ (nanoliters per millimeter per minute) was calculated from (12)

$J_{v}=V_{i}-V_{0} / L$

where $V_{i}$ and $V_{0}$ are the perfusion and collection rates, respectively (nanoliters per minute), and $L$ is the tubule length (millimeters). $L_{p}$ (centimeters per second per atmosphere) was determined by the formula (13)
$L_{p}=-\frac{V_{i} C_{i}}{R T A}\left[\frac{C_{i}-C_{0}}{C_{i} C_{0} C_{b}}+\frac{1}{\left(C_{b}\right)^{2}} \ln \frac{\left(C_{0}-C_{b}\right) C_{i}}{\left(C_{i}-C_{b}\right) C_{0}}\right]$

where $C_{i}, C_{0}$, and $C_{b}$ are the osmotic pressures of the perfusate, collected fluid, and bathing fluid, respectively (atmospheres), $R$ is the gas constant, $T$ is the temperature (degrees Kelvin), and $A$ is the tubular area (square centimeters), calculated from the measured tubule length and an assumed internal diameter of $20 \mu \mathrm{m}$ (for comparison with previously published observations $[14,15])$. The actual measured diameters of 11 sham and 11 UUO tubules were $30.1 \pm 5.3$ and $28.7 \pm 5.2 \mu \mathrm{m}$, respectively (means $\pm \mathrm{SD}$, values not significantly different by unpaired $t$ test). $C_{0}$ was calculated from the ratio of concentrations of tritiated inulin in the collected fluid and perfusate times $C_{i}(16,17)$. For measurement of $\mathrm{V}_{1}$, a saline-equilibrated $3 \mathrm{M} \mathrm{KCl}$ agar bridge was placed in contact with the bath (reference), and another bridge placed in contact with the tubule lumen through the perfusion pipette. The other ends of the agar bridges were in series with calomel half-cells connected to a high impedance electrometer (W-P Instruments, Inc., model 725, New Haven, CT). The transepithelial potential was continuously monitored on a strip chart recorder (model R511A, Beckman Instruments, Fullerton, CA).

The perfusion protocol consisted of three periods. During the first period (equilibration), the tubule was perfused for $90 \mathrm{~min}$ at $37^{\circ} \mathrm{C}$ and the $\mathrm{V}_{\mathrm{i}}$ adjusted to $7-10 \mathrm{nl} \cdot \mathrm{min}^{-1}$. During the second period (basal), three or four timed sequential samples of collected fluid were obtained in precalibrated volumetric pipettes of 40-50 $\mathrm{nl}$. The third period began with the addition to the bath of either $200 \mu \mathrm{U} \cdot \mathrm{ml}^{-1}$ arginine vasopressin (Pitressin, lot BB606, Parke-Davis, Morris Plains, NJ) or $10^{-4} \mathrm{M}$ 8-bromoadenosine 3',5'-cyclic monophosphate (8-Br-cAMP) (Sigma Chemical Co., St. Louis, MO). These concentrations of vasopressin or 8-Br-cAMP are known to produce maximal increases in water permeability in CCT (14-18). Sequential timed collections were continued after the addition of vasopressin or 8-Br-cAMP for a period of $90 \mathrm{~min}$. Each sample was counted for tritium in a liquid scintillation counter (Packard Instruments, Downer's Grove, IL) and the values of $V_{i}, J_{v}$, and $L_{p}$ were calculated. For data analysis each experiment was divided into a series of 10-min intervals, and mean values of $V_{i}, J_{v}$, and $L_{p}$ were calculated for each interval. These values were then used in calculating the means of each interval for the experimental group, and these values are shown in Results.

Animals were divided into four groups. Groups I and IV received no treatment before the experiment. Group II received desoxycorticosterone acetate (DOCA) (Sigma Chemical Co.) in sesame oil, $5 \mathrm{mg}$ intramuscularly daily, for 7-14 d prior to the study (14). Group III received indomethacin (Sigma Chemical Co.) dissolved in $0.1 \mathrm{M}$ Tris buffer, $5 \mathrm{mg} \cdot \mathrm{kg}^{-1}$ body wt intravenously, $30 \mathrm{~min}$ prior to the surgical procedure (sham operation or UUO) (19); indomethacin was present in the bath in these studies at $10^{-5} \mathrm{M}$. During the third period, tubules from groups I, II, and III were exposed to arginine vasopressin; tubules from group IV were exposed to 8-Br-cAMP.

In 11 tubules, lumen-to-bath sodium fluxes were measured isotopically. Animals were subjected to surgical procedures as detailed above. The tubules were perfused and bathed with identical isotonic solutions (the same as the bath solution that was previously described except for the substitution of $5 \mathrm{mM} \mathrm{Na}$ Hepes/20 mM Na cyclamate for the 25 $\mathrm{mM} \mathrm{NaHCO}, 3 \mathrm{pH} 7.4) .50 \mu \mathrm{Ci} / \mathrm{ml}$ of ${ }^{22} \mathrm{Na}$ were added to the perfusate solution in addition to tritiated inulin. $V_{i}$ 's in these tubules were adjusted to $\sim 2 \mathrm{nl} \cdot \mathrm{min}^{-1}$. Collected fluid was counted on a liquid scintillation counter with appropriate windows for determination of ${ }^{22} \mathrm{Na}$ and tritium counts. Under these conditions (no osmotic gradient), volume fluxes were not different from zero. The precise determination of lumen-to-bath sodium flux requires the measurement of the specific activity of the tracer in the collected fluid. However, minimum and maximum limits on the lumen-to-bath flux can be determined by assuming either zero backflux of unlabeled sodium (total luminal sodium concentration diminishes in proportion with the decline in tracer concentration) or backflux equal to lumen-to-bath flux (total 
luminal sodium concentration does not change). For comparison of lumen-to-bath sodium flux rates in tubules from sham-operated vs. UUO animals, the maximum possible value for the UUO group was compared with the minimum possible value for the sham-operated group.

Results shown are means \pm SE. The level of statistical significance was calculated using Student's paired or unpaired $t$ test, as required by the experimental design $(20,21)$.

\section{Results}

Fig. 1 shows the time course of vasopressin-induced changes in $L_{p}$ in tubules from animals in group I (no pretreatment) subjected to either sham surgery or $4 \mathrm{~h}$ of UUO. After $90 \mathrm{~min}$ of equilibration (in the absence of vasopressin), tubules from both sham-operated and UUO animals displayed low water permeability with values of $L_{p}$ which were not statistically different from zero. Thus, following UUO, tubules had retained their normally low $L_{p}$ and could maintain high osmotic gradients across the tubular epithelium. After addition of vasopressin $\left(200 \mu \mathrm{U} \cdot \mathrm{ml}^{-1}\right)$ to the bathing solution, tubules from UUO animals showed marked blunting of the $L_{p}$ response compared with tubules from sham-operated animals. Table I shows the mean values of $J_{v}$ and $L_{p}$ obtained in the basal period and in the $30-40-\mathrm{min}$ interval after the addition of vasopressin or 8-Br-cAMP (maximal response). $J_{v}$ and $L_{p}$ in the basal period were not different from zero in tubules from both sham-operated and UUO animals in all of the groups. After vasopressin or 8-Br-cAMP, $\mathrm{J}_{v}$ and $\mathrm{L}_{\mathrm{p}}$ increased in tubules from both sham-operated and UUO animals in all of the groups. However, $J_{v}$ and $L_{p}$ values in tubules from UUO animals after vasopressin or 8-Br-cAMP were smaller than the corresponding values observed in tubules from sham-operated animals. For group $I, L_{p}$ values in tubules from UUO animals

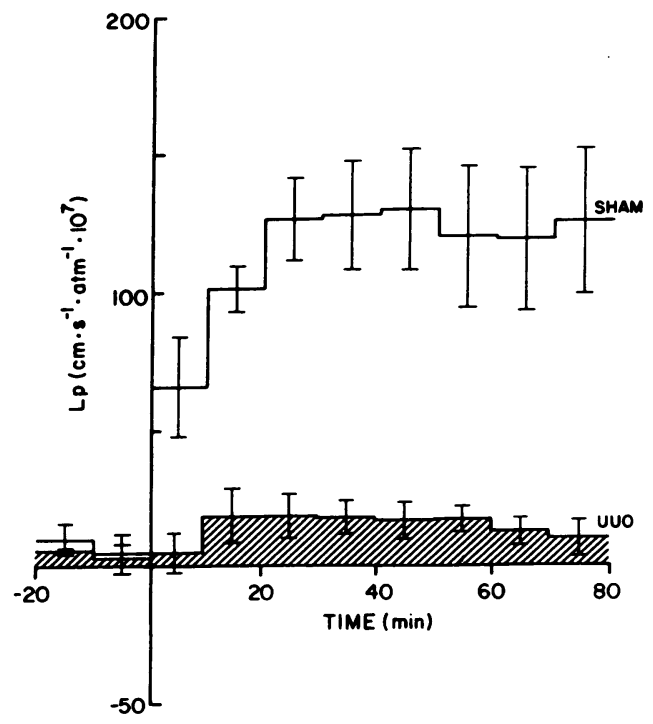

Figure 1. Time course of changes in $\mathrm{L}_{\mathrm{p}}$ in tubules from shamoperated (top) and UUO animals (bottom) in group I. Tubules were equilibrated at $37^{\circ} \mathrm{C}$ for $90 \mathrm{~min}$ before the first sampling interval. Arginine vasopressin was added at $t=0$ at a concentration of 200 $\mu \mathrm{U} \cdot \mathrm{ml}^{-1}$. Before the addition of vasopressin, basal $\mathrm{L}_{p}$ values were not different from zero in either group. Tubules from UUO animals developed $L_{p}$ responses that were significantly lower than those in tubules from sham-operated animals. were only $15 \%$ of the corresponding values in tubules from sham-operated animals.

Adrenal steroids have been shown to potentiate the $L_{p}$ response of CCT to vasopressin (14). To examine the effect of UUO on CCT from DOCA-treated animals, the studies in group II were done. The results are shown in Fig. 2. The $L_{p}$ in the basal period was not different from zero in tubules from either sham-operated or UUO animals; DOCA pretreatment enhanced the vasopressin response of tubules from shamoperated animals but marked suppression of the response was still observed in tubules from UUO animals. The values of $\mathbf{J}_{\mathbf{v}}$ and $L_{p}$ in the basal period and for the $30-40$-min period after vasopressin exposure are shown in Table I. Maximal vasopressin response of $\mathrm{L}_{p}$ was greater in the tubules from DOCA-treated animals than in tubules from animals that had not received DOCA treatment, for both the sham-operated groups (no pretreatment, 128 \pm 20 ; DOCA, 257 $\pm 49 ; P<0.05$ ) and for the UUO groups (no pretreatment, 19 \pm 6 ; DOCA, 46 $\pm 11 ; P$ $<0.05$ ). However, the $L_{p}$ value in tubules from DOCA-treated UUO animals was $18 \%$ of the value in tubules from DOCAtreated sham-operated animals, only slightly higher than the $15 \%$ value observed in group $\mathrm{I}$. The values of $\mathrm{J}_{\mathrm{v}}$ showed similar changes. Thus, UUO resulted in similar inhibition of the vasopressin-induced change in $L_{p}$ and $J_{v}$ in tubules from DOCA-treated animals as in tubules from animals that did not receive DOCA.

Prostaglandins have been shown to suppress vasopressininduced changes in $L_{p}$ in CCT $(22,23)$, and its synthesis by the kidney is augmented following UUO (7). To assess a possible role of prostaglandins in mediating the defect in vasopressin responsiveness of CCT after UUO, indomethacin was given before UUO. Fig. 3 shows the results of these experiments. Values of $\mathrm{L}_{p}$ in the absence of vasopressin were not different from 0 or from the other groups. Despite indomethacin pretreatment in vivo marked suppression of the vasopressin-induced response was seen again after UUO. Table I shows the values of $J_{v}$ and $L_{p}$ for the periods before and after vasopressin exposure. Absolute values for the peak response for $J_{v}$ and $L_{p}$ in the tubules from indomethacin-treated UUO animals were not significantly different from the corresponding values in group $I$. The peak response for $\mathrm{L}_{p}$ in tubules from indomethacin-treated UUO animals was $26 \%$ of the value in the tubules from indomethacin-treated sham-operated animals.

To test the possibility that UUO induces a defect in the vasopressin-receptor adenylate-cyclase complex, a cAMP analogue (8-Br-cAMP) was used. The results of these experiments are shown in Fig. 4 and Table I. The 8-Br-cAMP-induced increase in $J_{v}$ and $L_{p}$ in tubules from sham-operated animals was not statistically different from the increase induced by vasopressin in group I. UUO suppressed the water permeability response of CCT to 8-Br-cAMP. $\mathrm{L}_{\mathrm{p}}$ values in tubules from UUO animals at $30-40 \mathrm{~min}$ after $8-\mathrm{Br}$-cAMP addition were $29 \%$ of the values in tubules from the sham-operated animals during the same interval.

The results of the $V_{1}$ measurements (determined before the addition of vasopressin or 8-Br-cAMP) are shown in Table II. Under the conditions of these experiments (low perfusate sodium), tubules from sham-operated animals that received no pretreatment (groups I and IV) developed mean voltages of $-22+4 \mathrm{mV}$ (lumen negative). Tubules from UUO animals developed much lower $\mathrm{V}_{1}(-5 \pm 2 \mathrm{mV}, P<0.005$ compared 
Table I. $J_{v}$ and $L_{p}$ in CCT from Sham-operated Animals and Animals with UUO

\begin{tabular}{|c|c|c|c|c|c|c|c|}
\hline \multirow[b]{2}{*}{ Group } & & \multicolumn{2}{|c|}{$\underline{\mathrm{J}_{v}\left(n I \cdot m m^{-1} \cdot \min ^{-1}\right)}$} & \multicolumn{2}{|c|}{$\mathrm{L}_{\mathrm{p}}\left(\mathrm{cm} \cdot \mathrm{s}^{-1} \cdot a t m^{-1} \cdot 10^{7}\right)$} & \multicolumn{2}{|l|}{$\underline{n}$} \\
\hline & & Sham & uUo & Sham & uUo & Sham & UUo \\
\hline I & $\begin{array}{l}\text { Basal } \\
\text { aVP }\end{array}$ & $\begin{array}{l}0.07 \pm 0.11 \\
1.64 \pm 0.23^{*}\end{array}$ & $\begin{array}{l}0.05 \pm 0.08 \\
0.27 \pm 0.09 * \ddagger\end{array}$ & $\begin{array}{c}4.6 \pm 7.3 \\
127.9 \pm 19.8^{*}\end{array}$ & $\begin{array}{c}3.6 \pm 5.4 \\
18.8 \pm 6.2^{*} \ddagger\end{array}$ & 7 & 7 \\
\hline II & $\begin{array}{l}\text { Basal } \\
\text { aVP }\end{array}$ & $\begin{array}{l}0.07 \pm 0.04 \\
2.92 \pm 0.56^{*}\end{array}$ & $\begin{array}{l}0.08 \pm 0.03 \\
0.67 \pm 0.18^{*} \ddagger\end{array}$ & $\begin{array}{c}5.1 \pm 2.1 \\
257.1 \pm 49.4^{*}\end{array}$ & $\begin{array}{c}5.4 \pm 1.9 \\
46.3 \pm 11.8^{*} \ddagger\end{array}$ & 6 & 4 \\
\hline III & $\begin{array}{l}\text { Basal } \\
\text { aVP }\end{array}$ & $\begin{array}{r}-0.02 \pm 0.08 \\
1.70 \pm 0.29 *\end{array}$ & $\begin{array}{l}0.02 \pm 0.04 \\
0.52 \pm 0.14^{*} \ddagger\end{array}$ & $\begin{array}{l}-2.3 \pm 6.3 \\
141.5 \pm 24.6^{*}\end{array}$ & $\begin{array}{c}1.3 \pm 2.9 \\
37.1 \pm 10.4^{*} \ddagger\end{array}$ & 5 & 5 \\
\hline IV & $\begin{array}{l}\text { Basal } \\
\text { 8-Br-cAMP }\end{array}$ & $\begin{array}{l}0.11 \pm 0.09 \\
2.04 \pm 0.13^{*}\end{array}$ & $\begin{array}{l}0.05 \pm 0.05 \\
0.87 \pm 0.17^{*} \ddagger\end{array}$ & $\begin{array}{c}7.1 \pm 6.0 \\
187.9 \pm 28.3^{*}\end{array}$ & $\begin{array}{c}3.4 \pm 3.4 \\
54.9 \pm 12.9^{*} \ddagger\end{array}$ & 5 & 5 \\
\hline
\end{tabular}

aVP, arginine vasopressin. Values are means \pm SE. $n$, number of tubules studied. $* P<0.05$ for $J_{v}$ or $L_{p}$ values after aVP or 8-Br-cAMP value compared with basal. $\ddagger P<0.05$ for $\mathrm{J}_{v}$ or $\mathrm{L}_{\mathrm{p}}$ (aVP- or 8-Br-cAMP-stimulated UUO values) compared with sham-operated values.

with tubules from the corresponding sham-operated animals). Tubules from sham-operated DOCA-pretreated animals developed mean $V_{1}(-43 \pm 7 \mathrm{mV})$ that were higher than $V_{1}$ in tubules from sham-operated animals in groups I and IV, in accordance with values reported in the literature (24). Tubules from DOCA-treated UUO animals developed lower voltages $(-3 \pm 2$ $\mathrm{mV})$ than tubules from either the DOCA-treated shams $(P$ $<0.005)$ or the nontreated shams $(P<0.005)$, but the voltages in tubules from the DOCA-treated UUO animals were not significantly different from the voltages in tubules from nontreated UUO animals. Tubules from indomethacin-treated sham-operated animals developed voltages of $-10 \pm 4 \mathrm{mV}$. Tubules from indomethacin-treated UUO animals developed higher voltages $(-17 \pm 1 \mathrm{mV})$ than the other UUO groups. The voltages in tubules from indomethacin-treated UUO animals were not significantly different from the voltages in tubules from the indomethacin-treated sham-operated animals or from voltages in tubules from the sham-operated animals in groups I and IV (no pretreatment). The voltages in tubules from the

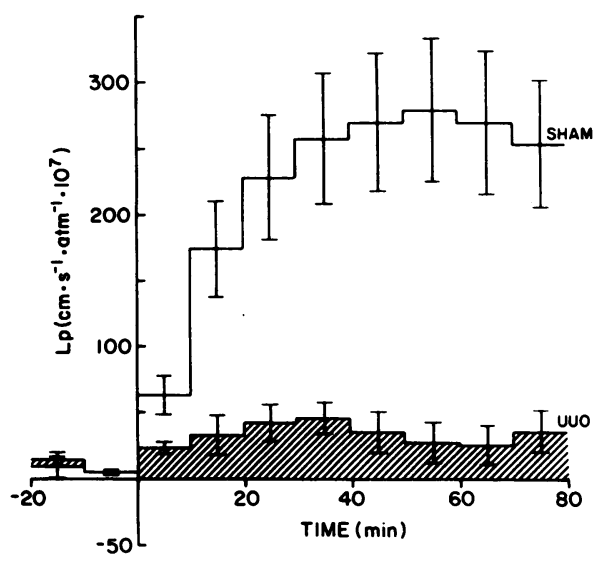

Figure 2. Time course of changes in $\mathrm{L}_{\mathrm{p}}$ in tubules from shamoperated (top) and UUO animals (bottom) in group II (5 mg DOCA intramuscularly for 10-14 d before study). Note the change in scale. The perfusion protocol is as in Fig. 1. DOCA treatment increased the $L_{p}$ response in both sets of tubules, but the relative degree of suppression for the UUO animals was similar to that in group I. indomethacin-treated UUO animals were higher than those in tubules from the other UUO groups (vs. groups I and IV, $P$ $<0.02$; vs. group II, $P<0.05$ ). Thus, UUO caused a defect in the ability of the CCT to develop a lumen-negative voltage when perfused at $37^{\circ} \mathrm{C}$ in vitro, and the defect was prevented by treatment of the animal with indomethacin before UUO.

To test the hypothesis that the low voltages measured in the UUO tubules reflect low rates of sodium transport, isotopic flux measurements were done in a separate series of tubules as described above. These tubules were exposed to identical solutions in the perfusate and bath (no sodium or osmotic gradients). In six tubules taken from animals subjected to sham surgery, the minimum possible lumen-to-bath sodium flux was $38.2 \pm 4.0 \mathrm{pmol} \cdot \mathrm{mm}^{-1} \cdot \mathrm{min}^{-1}$. In five tubules taken from animals with UUO, the maximum possible lumen-tobath sodium flux was $26.2 \pm 3.3 \mathrm{pmol} \cdot \mathrm{mm}^{-1} \cdot \mathrm{min}^{-1}$. These values are significantly different $(P<0.05)$. Thus, obstruction depresses lumen-to-bath sodium fluxes in CCT.

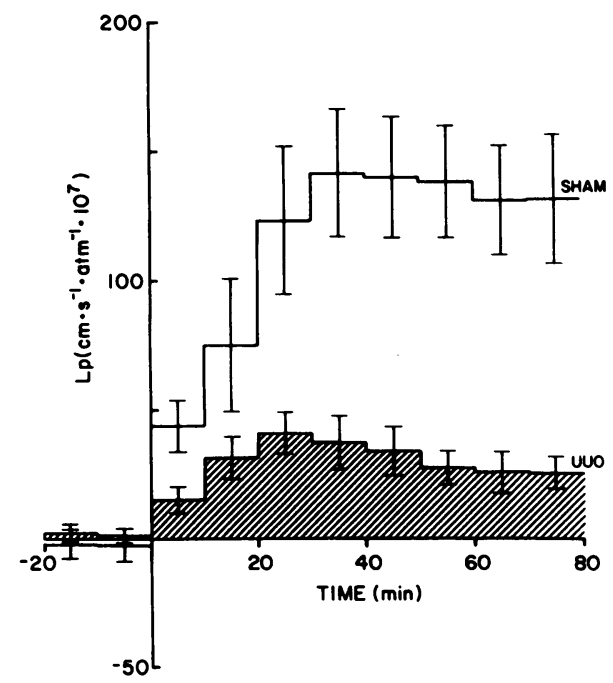

Figure 3. Time course of changes in $\mathrm{L}_{p}$ in tubules from shamoperated (top) and UUO animals (bottom) in group III $\left(5 \mathrm{mg} \cdot \mathrm{kg}^{-1}\right.$ indomethacin intravenously $30 \mathrm{~min}$ before sham operation or UUO). The perfusion protocol is as in Fig. 1. 


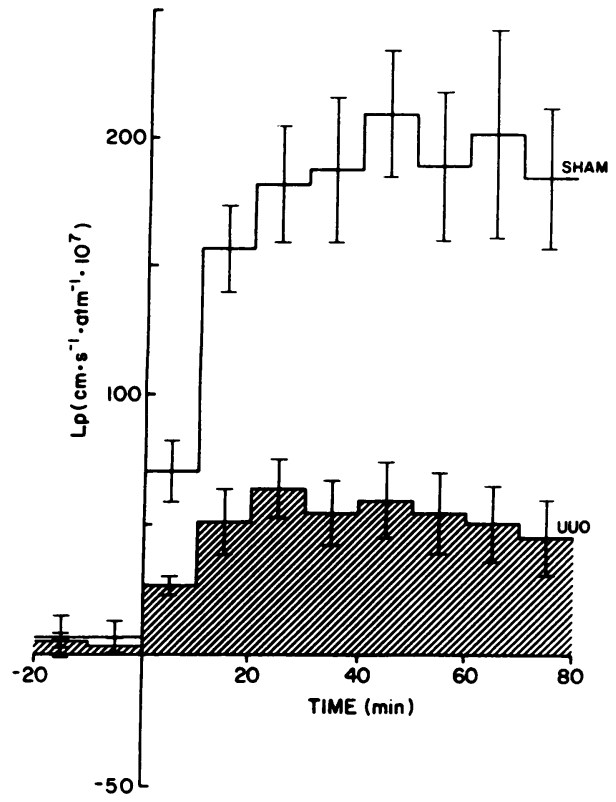

Figure 4. Time course of changes in $L_{p}$ in tubules from shamoperated (top) and UUO animals (bottom) in group IV. The perfusion protocol is as in Fig. 1, except that $10^{-4} \mathrm{M}$ 8-Br-cAMP was added at $t=0$ instead of vasopressin.

\section{Discussion}

Our results demonstrate intrinsic defects in the cortical collecting duct following a brief period of UUO. We showed two separate abnormalities: resistance to the hydroosmotic action of arginine vasopressin and failure to develop $V_{1}$ comparable with control tubules. These data may help to explain some of the abnormalities noted after the release of ureteral obstruction or during partial ureteral obstruction.

Hanley and Davidson (8), utilizing the isolated perfused tubule technique, reported similar abnormalities in rabbit tubules after $24 \mathrm{~h}$ of bilateral or UUO. In their experiments, cortical collecting duct function was assessed by bathing the tubules in vasopressin or CAMP with isotonic solutions in the bath and lumen and suddenly raising bath osmolality to hypertonic levels by the addition of raffinose. Obstructed tubules from both unilaterally and bilaterally obstructed kidneys had lower $L_{p}$ than tubules from normal kidneys. The $L_{p}$ of the tubules in the absence of vasopressin was not assessed.

Table II. $V_{1}$ in CCT from Sham-operated Animals and Animals with UUO

\begin{tabular}{llllll}
\hline & $V_{1}(m V)$ & & & $n$ \\
\cline { 2 - 3 } \cline { 5 - 6 } Group & Sham & UUO & & Sham & UUO \\
\hline I + IV & $-21.9 \pm 4.1$ & $-5.0 \pm 2.1^{*}$ & & 12 & 12 \\
II & $-42.8 \pm 7.2$ & $-3.4 \pm 1.7^{*}$ & & 6 & 4 \\
III & $-9.8 \pm 3.6$ & $-17.3 \pm 1.7 \ddagger$ & 5 & 5 \\
\hline
\end{tabular}

Values are means $\pm \mathrm{SE}$ measured during the basal period.

* $P<0.05$, UUO vs. sham-operated in the same group.

¥ NS compared with sham-operated animals in groups I, III, or IV.
Our data indicate that the CCT epithelium can maintain low water permeability in the absence of vasopressin despite UUO (that is, the epithelium remains "tight" with respect to water). UUO does induce a defect in this epithelium with respect to water transport, specifically, the inability to increase $L_{p}$ in response to vasopressin.

The decreased response to vasopressin was present even though our study used a much shorter period of obstruction $(4 \mathrm{~h})$ than that of Hanley and Davidson (8). This defect was demonstrable with the tubule perfused in vitro, and thus was not due to the effects of changes in fluid delivery to the collecting duct or to changes in the osmotic gradients present. Also, the defect was intrinsic to the collecting duct itself and was not due to constant exposure to a factor or factors produced elsewhere that would mediate changes in epithelial vasopressin responsiveness. Finally, physical factors cannot account for the defect since the perfusion conditions between the obstructed and control tubules were the same.

From our results, the mechanism by which obstruction affects vasopressin responsiveness is not clear. The cellular events that result in changes in $L_{p}$ in vasopressin-sensitive epithelia begin with the binding of the hormone to a receptor on the basolateral membrane of the cell, with activation of adenylate cyclase and intracellular generation of cAMP (25). To test the hypothesis that a defect in this hormone receptor/ cyclase complex occurs as a result of obstruction, we used an analogue of CAMP to bypass hormone binding and cAMP generation. The $L_{p}$ of tubules from UUO animals exposed to 8-Br-cAMP was significantly less than the $L_{p}$ observed in tubules from sham-operated controls. This result agrees with that obtained by Hanley and Davidson (8) in their model of obstruction, and indicates that the major defect in vasopressin responsiveness is not due to altered interactions of the hormone with its receptor or the subsequent activation of adenylate cyclase. We cannot rule out the possibility that a defect in the hormone receptor or in the coupling of the receptor to adenylate cyclase is present but masked by another abnormality distal to cAMP generation. In fact, the fractional response (that is, the response in the tubules from UUO animals expressed as a fraction of the response in corresponding tubules from sham-operated animals) of the 8-Br-cAMP-exposed tubules was about twice that of the tubules exposed to vasopressin (group I), a finding consistent with the thesis that the defect is partially related to abnormalities in the hormone receptor/ cyclase complex. Further studies are needed to investigate this possibility. A similar defect for vasopressin responsiveness has been suggested for the cortical collecting duct from the remnant kidney of rabbits with uremia (26).

Mineralocorticoids act to enhance the hydroosmotic responsiveness of vasopressin-sensitive epithelia (14), thus playing a role in regulating the water permeability response in the collecting duct. Batlle, Arruda, and Kurtzman (27) reported resistance to the effects of mineralocorticoids on urinary acidification and potassium excretion in patients with ureteral obstruction. We decided to test whether or not DOCA would enhance the change in $\mathrm{L}_{p}$ in response to vasopressin in the tubules from UUO animals and whether DOCA pretreatment would offer protection against the effects of obstruction beyond the normal enhancement. The absolute value of $L_{p}$ was significantly greater in tubules from DOCA-pretreated UUO animals than in tubules from nontreated UUO animals, indicating that DOCA enhances vasopressin responsiveness despite 
UUO. When expressed as a percent of the control response, the $\mathrm{L}_{\mathrm{p}}$ in tubules from nontreated UUO animals was $15 \%$ and in tubules from DOCA-treated animals, $18 \%$. Thus, DOCA pretreatment enhances vasopressin responsiveness but does not offer protection against the effects of obstruction.

It is known that prostaglandins of the $\mathrm{E}$ series blunt the vasopressin response of normal CCT $(22,23)$, and that prostaglandin E synthetic rates are greatly increased following UUO (28). It has been suggested that the abnormal response of the CCT following obstruction is a result of this excessive prostaglandin synthesis (2). Inhibition of the prostaglandin system with indomethacin had no demonstrable protective effect on the hydroosmotic response of the tubule to vasopressin despite its effects on voltage (see below). Although enhanced prostaglandin synthesis might be important in other ways, such as effects on the thick ascending limb, alterations in medullary blood flow, or distal delivery of solute and water (2), our studies show that contributions of the prostaglandin system do not fully explain the defects in cortical collecting duct responsiveness to vasopressin.

Our data indicate that the major abnormality in vasopressin responsiveness of the CCT following UUO lies at a step distal to cAMP generation and is not due to resistance to the permissive effects of mineralocorticoids or to an effect of enhanced prostaglandin synthesis. A number of other factors are known to alter vasopressin responsiveness, such as calcium and calmodulin $(16,17)$, kinins (29), and intracellular $\mathrm{pH}$ (30). It is possible that alterations in one or more of these factors is responsible for the abnormality in vasopressin responsiveness. The defect could relate to the availability of preformed water channels or the mechanisms by which the water channels are inserted into the membrane (31). Finally, the defect could reside in the mechanism by which bulk water flow is directed across the cell (32).

In our study, tubules taken from animals subjected to $4 \mathrm{~h}$ of UUO did not develop lumen-negative voltages' as high as did tubules from sham-operated animals. Further, when lumento-bath sodium transport was assessed, tubules from UUO animals had lower sodium fluxes than did tubules taken from sham-operated animals. Thus, it appears that the $V_{1}$ in the obstructed tubules parallels the rate of lumen-to-bath sodium transport, as has been reported for nonobstructed CCT by others (14). The finding of depressed sodium transport by this segment may help explain the abnormalities seen in renal sodium conservation after ureteral obstruction, since the function of the cortical collecting duct is important in the regulation of sodium excretion. Maneuvers which stimulate production of endogenous mineralocorticoids or the administration of exogenous mineralocorticoids stimulate sodium reabsorption and potassium secretion and result in higher voltages in the in vitro perfused collecting tubule (14). Our results in the shamoperated animals agree with these observations in regard to the voltages. Despite the effects of DOCA in tubules from sham-operated animals, DOCA did not increase voltage in the tubules taken from animals subjected to $4 \mathrm{~h}$ of ureteral obstruction with $V_{1}$ values being no different than those seen in tubules taken from UUO animals not given mineralocorticoid pretreatment. As in the nontreated group, the low voltage probably reflects lower rates of sodium transport and may explain the site of mineralocorticoid resistance described by Batlle, Arruda, and Kurtzman (27).

In contrast to the effects of obstruction on voltage in tubules taken from animals treated with DOCA or from animals with no treatment, tubules taken from animals treated with indomethacin developed voltages that were not different from tubules from sham-operated animals. This result strongly suggests that the decrease in voltage observed in tubules from UUO kidneys is mediated by enhanced prostaglandin synthesis, which may cause a decrease in sodium transport by the CCT. In normal CCT, prostaglandins depress sodium transport (33, 34). The results in this group show that the two defects induced by obstruction that we studied are separable, one being preventable by administration of indomethacin and the other not.

In summary, we have demonstrated that following UUO of 4-h duration defects occur in the ability of the cortical collecting duct to respond to vasopressin with normal increases in $L_{p}$ and in the ability to generate normal $V_{1}$ 's. The defect in vasopressin responsiveness was not a result of enhanced prostaglandin synthesis, and could not be prevented by pretreatment with mineralocorticoids. The vasopressin-receptor adenylate cyclase system was not the major site of the defect. The defect in the ability to generate $V_{1}$ 's was associated with low rates of lumen-to-bath sodium transport. The defect in the ability to generate $V_{1}$ 's could be prevented by indomethacin treatment. Although we did not measure sodium fluxes in CCT from obstructed kidneys after pretreatment with indomethacin, the results described above suggest that enhanced prostaglandin synthesis may result in depression of sodium transport. The defects in both $L_{p}$ and $V_{1}$ are intrinsic to the postobstructed CCT and indicate that changes in the epithelium per se are involved in the abnormalities in renal sodium and water handling that occur as a result of obstruction.

\section{Acknowledgments}

The secretarial assistance of Sue Eads is gratefully acknowledged.

This study was supported in part by the National Institutes of Health Research grant P01 AM09976-19 and by National Institute of Health Training grant AM07126.

\section{References}

1. Badr, K., I. Ichikawa, and B. M. Brenner. 1983. Renal circulatory and nephron function in experimental obstruction of the urinary tract. In Acute Renal Failure. B. M. Brenner and J. M. Lazarus, editors. W. B. Saunders, Philadelphia, PA. 116-136.

2. Klahr, S. 1983. Pathophysiology of obstructive nephropathy. Kidney Int. 23:414-426.

3. Muldowney, F. P., C. J. Duffy, D. G. Kelly, F. A. Duff, D. Harrington, and R. Freany. 1966. Sodium diuresis after relief of obstructive uropathy. N. Engl. J. Med. 274:1294-1298.

4. Wilson, D. R., and U. Honrath. 1976. Cross-circulation study of natriuretric factors in post-obstructive diuresis. J. Clin. Invest. 57:380-389.

5. Moody, T. E., E. D. Vaughn, and J. Y. Gillenwater. 1975. Relationship between renal blood flow and ureteral pressure during 18 hours of total ureteral occlusion. Implications for changing sites of increased renal resistance. Invest. Urol. 13:246-251.

6. Wright, F. S. 1982. Effects of urinary tract obstruction on glomerular filtration rate and renal blood flow. Sem. Nephrol. 2:5-16.

7. Morrison, A. R. 1982. Alterations in intrarenal hormones in urinary tract obstruction. Sem. Nephrol. 2:40-45.

8. Hanley, M. J., and K. Davidson. 1982. Isolated nephron segments from rabbit models of obstructive nephropathy. J. Clin. Invest. 69:165-174.

9. Kaissling, B., and W. Kriz. 1979. Structural Analysis of the Rabbit Kidney. Springer-Verlag, Berlin. 89-100. 
10. Burg, M., J. J. Grantham, M. Abramow, and J. Orloff. 1966. Preparation and study of fragments of single rabbit nephron. Am. J. Physiol. 210:1293-1298.

11. Bello-Reuss, E. 1982. Electrical properties of the basolateral membrane of the straight portion of the rabbit proximal renal tubule. J. Physiol. 326:49-63.

12. Bello-Reuss, E., Y. Higashi, and Y. Kaneda. 1982. Dopamine decreases fluid reabsorption in straight portions of rabbit proximal tubule. Am. J. Physiol. 242:F634-F640.

13. DuBois, R., A. Verniory, and M. Abramow. 1976. Computation of the osmotic water permeability of perfused tubule segments. Kidney Int. 10:478-479.

14. Schwartz, M. J., and J. P. Kokko. 1980. Urinary concentrating defect of adrenal insufficiency. J. Clin. Invest. 66:234-242.

15. Hall, D. E., and J. J. Grantham. 1980. Temperature effect on ADH response of isolated perfused rabbit collecting tubules. Am. J. Physiol. 239:F595-F601.

16. Goldfarb, S. 1982. Effects of calcium on ADH action in the cortical collecting tubule perfused in vitro. Am. J. Physiol. 243:F481F486.

17. Frindt, G., E. E. Windhager, and A. Taylor. 1982. Hydrosmotic response of collecting tubules to ADH or CAMP at reduced peritubular sodium. Am. J. Physiol. 243:F503-F513.

18. Grantham, J. J., and M. B. Burg. 1966. Effect of vasopressin and cyclic AMP on permeability of isolated perfused rabbit collecting tubules. Am. J. Physiol. 239:F595-F601.

19. Campbell, W. B., E. K. Jackson, and R. M. Graham. 1979. Saralasin-induced renin release: its blockade by prostaglandin synthesis inhibitors in the conscious rat. Hypertension. 1:637-642.

20. Swinscow, T. D. V. 1980. Statistics at Square One. The Mendip Press, Bath, England. 1-86.

21. Diem, K., and C. Lentner. 1970. Documenta Geigy: Scientific Tables, Ucclesfield, Geigy Pharmaceuticals. Seventh ed. 32.

22. Kirschenbaum, M. A., A. G. Lowe, W. Trizna, and L. G. Fine. 1982. Regulation of vasopressin action by prostaglandins. J. Clin. Invest. 70:1193-1204.
23. Grantham, J. J., and J. Orloff. 1968. Effect of prostaglandin E on the permeability response of the isolated collecting tubule to vasopressin, adenosine $3^{\prime}, 5^{\prime}$-monophosphate and theophylline. J. Clin. Invest. 47:1154-1161.

24. Stokes, J. B., M. J. Ingram, A. D. Williams, and D. Ingram. 1980. Heterogeneity of the rabbit collecting tubule: localization of the mineralocorticoid hormone action to the cortical portion. Kidney Int. 20:340-347.

25. Orloff, J. 1981. Regulation of cyclic AMP (cAMP) metabolism in water transport across epithelia. Alfred Benzon Symposium, Volume 15. Munksgaard, Copenhagen. 393-401.

26. Fine, L. G., D. Schlondorf, W. Trizna, R. M. Gilbert, and N. S. Bricker. 1978. Functional profile of the isolated uremic nephron. J. Clin. Invest. 60:1519-1527.

27. Batlle, D. C., J. A. L. Arruda, and N. A. Kurtzman. 1981. Hyperkalemic distal renal tubular acidosis associated with obstructive uropathy. N. Engl. J. Med. 304:373-380.

28. Morrison, A. R., H. Moritz, and P. Needleman. 1978. Mechanism of enhanced renal prostaglandin biosynthesis in ureteral obstruction: role of de novo protein synthesis. J. Biol. Chem. 253:8210-8218.

29. Schuster, U. L., J. P. Kokko, and H. R. Jacobson. 1984. Interactions of bradykinin and antidiuretic hormone in the cortical collecting tubule. Kidney Int. 25:315. (Abstr.)

30. Lorenzen, M., A. Taylor, and E. E. Windhager. 1983. pH Effect on osmotic response of collecting tubules to vasopressin and 8CPT-cAMP. Am. J. Physiol. 245:F188-F197.

31. Hays, R. M. 1983. Alteration of luminal membrane structure by antidiuretic hormone. Am. J. Physiol. 245:C289-C296.

32. DiBona, D. R. 1983. Cytoplasmic involvement in ADHmediated osmosis across toad urinary bladder. Am. J. Physiol. 245:C297C307.

33. Holt, W. F., and C. Lechene. 1981. ADH-PGE ${ }_{2}$ interactions in cortical collecting tubule. I. Depression of sodium transport. Am. J. Physiol. 241:F452-F460.

34. Kokko, J. P. 1981. Effect of prostaglandins on renal epithelial electrolyte transport. Kidney Int. 19:791-796. 